\title{
Equações Volumétricas e Fator de Forma e de Casca para Florestas Secundárias do Litoral de Santa Catarina
}

\author{
Jean Correia ${ }^{1}$, Alfredo Fantini ${ }^{1}$, Geferson Piazza ${ }^{1}$ \\ ${ }^{1}$ Departamento de Fitotecnia, Universidade Federal de Santa Catarina - UFSC, Florianópolis/SC, Brasil
}

\begin{abstract}
RESUMO
As florestas secundárias, predominantes em Santa Catarina, apresentam alto potencial para produção sustentável de madeira, entretanto faltam informações para estimar sua produtividade. Este estudo teve o objetivo de gerar equações de volumes de fuste e total (incluindo galhos), além dos fatores de forma e de casca, para florestas secundárias da Floresta Ombrófila Densa do Estado. Foram cubadas 187 árvores pelo método de Smalian e testados seis modelos volumétricos, utilizando-se análise de regressão, todos com resultados satisfatórios, destacando-se o de Schumacher-Hall (linearizado). As equações recomendadas para estimar o volume total com e sem casca são, respectivamente, $\ln (\mathrm{vt})=-10,045586+2,349493^{*} \ln (\mathrm{dap})+0,640598^{*} \ln (\mathrm{ht})$ e $\ln (\mathrm{vt})=-10,206982+2,348857^{*} \ln (\mathrm{dap})+0,634736^{*} \ln (\mathrm{ht})$, e para os volumes do fuste com e sem casca, respectivamente, $\ln (\mathrm{vf})=-9,285656+2,100113^{*} \ln ($ dap $)+0,665269^{*} \ln (\mathrm{hf})$ e $\ln (\mathrm{vf})=-9,504432+2,140098^{*} \ln (\mathrm{dap})+0,637631^{*} \ln (\mathrm{hf})$. O fator de forma normal estimado é 0,8027 , enquanto o fator de casca é 0,9301 .
\end{abstract}

Palavras-chave: volumetria, Mata Atlântica, dendrometria e inventário florestal.

\section{Volume Equations and Form and Bark Factors for Coastal Secondary Forests of Santa Catarina State}

\begin{abstract}
Secondary forests prevalent is Santa Catarina State present high potential for sustainable production of timber. However, we lack information to estimate their productivity. This study aimed at generating equations to estimate the stem volume and total volume (including branches), in addition to the form and bark factors, for secondary forests of the Atlantic rainforest in the State. We scaled 187 trees by Smalian method and tested six volumetric models through regression analysis, all with satisfactory results, notably the Schumacher-hall (linearized). The recommended equations to estimate the total volume with and without bark are, respectively, $\ln (\mathrm{vt})=-10.045586+2.349493^{\star} \ln (\mathrm{dap})+0.640598^{\star} \ln (\mathrm{ht})$ and $\ln (\mathrm{vt})=-10.206982+2.348857^{*} \ln (\mathrm{dap})+0.634736^{*} \ln (\mathrm{ht})$, and to estimate the stem volume with and without bark, respectively, $\ln (\mathrm{vf})=-9.285656+2.100113^{*} \ln (\mathrm{dap})+0.665269^{*} \ln (\mathrm{hf})$ and $\ln (\mathrm{vf})=-9,504432+2.140098^{\star} \ln (\mathrm{dap})+0.637631^{\star} \ln (\mathrm{hf})$. The estimated form factor is 0.8027 , while the bark factor is 0.9301 .
\end{abstract}

Keywords: volume modeling, Atlantic forest, dendrometry and forest inventory. 


\section{INTRODUÇÃO}

O histórico de má utilização dos recursos florestais e a substituição das florestas por outros usos da terra na região da Mata Atlântica são bem documentados (Dean, 1996), mas essa compreensão não tem evitado que o bioma continue perdendo grande parte da cobertura vegetal remanescente, como revelam dados recentes sobre o desmatamento (Fundação SOS Mata Atlântica, 2013). Em parte, o fenômeno pode ser explicado pela forte restrição ao manejo das florestas imposta pela "Lei da Mata Atlântica", que levou muitos proprietários rurais a converter suas terras em plantios homogêneos de espécies florestais exóticas, com consequente perda de biodiversidade (Siminski \& Fantini, 2010; Siminski et al., 2011b). A estratégia de restrição do uso dos recursos vem na contramão de forte movimento em favor da conservação pelo uso, que ganha espaço como proposta de conservação de florestas tropicais em todo o mundo (Redford \& Richter, 1999; Barrance et al., 2009). A conservação pelo uso seria conveniente na região do bioma Mata Atlântica, onde predominam os pequenos agricultores, os quais poderiam manejar suas florestas secundárias como fonte alternativa de renda. Em Santa Catarina, estado com $29 \%$ de cobertura florestal original, esse tipo de ecossistema é predominante (Vibrans et al., 2012a), havendo florestas jovens, com alta produtividade e potencial para produzir madeiras de boa qualidade, além de outros produtos florestais (Fantini \& Siminski, 2011).

A realização desse potencial depende de planejamento adequado. A determinação do volume de estoque de madeiras comerciais, por exemplo, é uma das informações básicas para planejar o manejo, visando a um correto ordenamento e dimensionamento da produção em níveis sustentáveis. O custo de obtenção dessa variável em florestas nativas é alto, mas pode ser reduzido com o uso de modelos matemáticos que permitem estimá-la, medindo-se variáveis de mais simples obtenção, como o diâmetro à altura do peito (DAP) (Silva \& Paula, 1979; Finger, 1992).

A maioria dos estudos de volumetria de espécies nativas se restringe à Amazônia e ao Cerrado, pela possibilidade de manejo nesses biomas. Os estudos relacionados à Mata Atlântica estão restritos ao estado de São Paulo (SP) (em geral, florestas plantadas), e alguns relatos são descritos para o Paraná (PR), Rio Grande do Sul (RS) e Espírito Santo (ES). Destacam-se os relacionados à Floresta Ombrófila Densa (FOD) no ES (Jorge, 1982; Souza \& Jesus, 1991; Chichorro et al., 2003) e em SP (Nunes, 2013). Alguns outros exemplos para a Mata Atlântica são relatados por Scolforo et al. (1994) em estudos de Floresta Estacional Semidecidual (FES) de MG, Santos et al. (2009) para Floresta Ombrófila Mista (FOM) no PR, Borsoi (2005) para FOM do RS e por Durlo \& Denardi (2009) para Cabralea canjerana em Floresta Estacional Decidual (FED) do RS.

Para Santa Catarina, há poucas publicações, sendo o mais recente estudo publicado no Inventário Florístico Florestal (Vibrans et al., 2012b) referente às principais formações do estado. Para a FOD, os poucos relatos são de estudos específicos para Miconia cinnamomifolia (Schuch et al., 2008) e Tibouchina pulchra (Oller, 2011), além de equações gerais (Silveira, 2008).

Nesse contexto, o presente estudo teve o objetivo de gerar equações de volumes de fuste e total das árvores, com e sem casca, além de fatores de forma e de casca, para florestas secundárias da FOD de Santa Catarina (SC). Os dados foram coletados de árvores abatidas em um projeto de pesquisa sobre o manejo de florestas secundárias. Trata-se, portanto, de uma oportunidade rara de coleta de dados com grande confiabilidade.

\section{MATERIAL E MÉTODOS}

\section{1. Área de estudo e coleta de dados}

A floresta estudada possui 42 ha de extensão e localiza-se no município de Guaramirim (SC) (Figura 1), local de execução de um projeto de pesquisa em manejo de florestas secundárias para produção de madeira, uma parceria entre universidades e a Fundação do Meio Ambiente de Santa Catarina (Fatma).

A formação florestal original do local é do tipo Floresta Ombrófila Densa (Klein, 1978), classificada como submontana conforme o Instituto Brasileiro de Geografia e Estatística (IBGE, 2012). O clima da região é do Tipo Cfa - temperado úmido -, com verões quentes e sem estação seca definida, segundo a classificação de Köppen. O relevo é acidentado, com declividades entre $10 \%$ e $40 \%$, e a altitude média é de 300 metros acima do nível do mar.

Foram cubadas 187 árvores de 40 diferentes espécies, com DAP entre 5,3 e $55,4 \mathrm{~cm}$. O conjunto de árvores cubadas é típico das florestas secundárias da região, tanto em termos de diversidade de espécies como da 


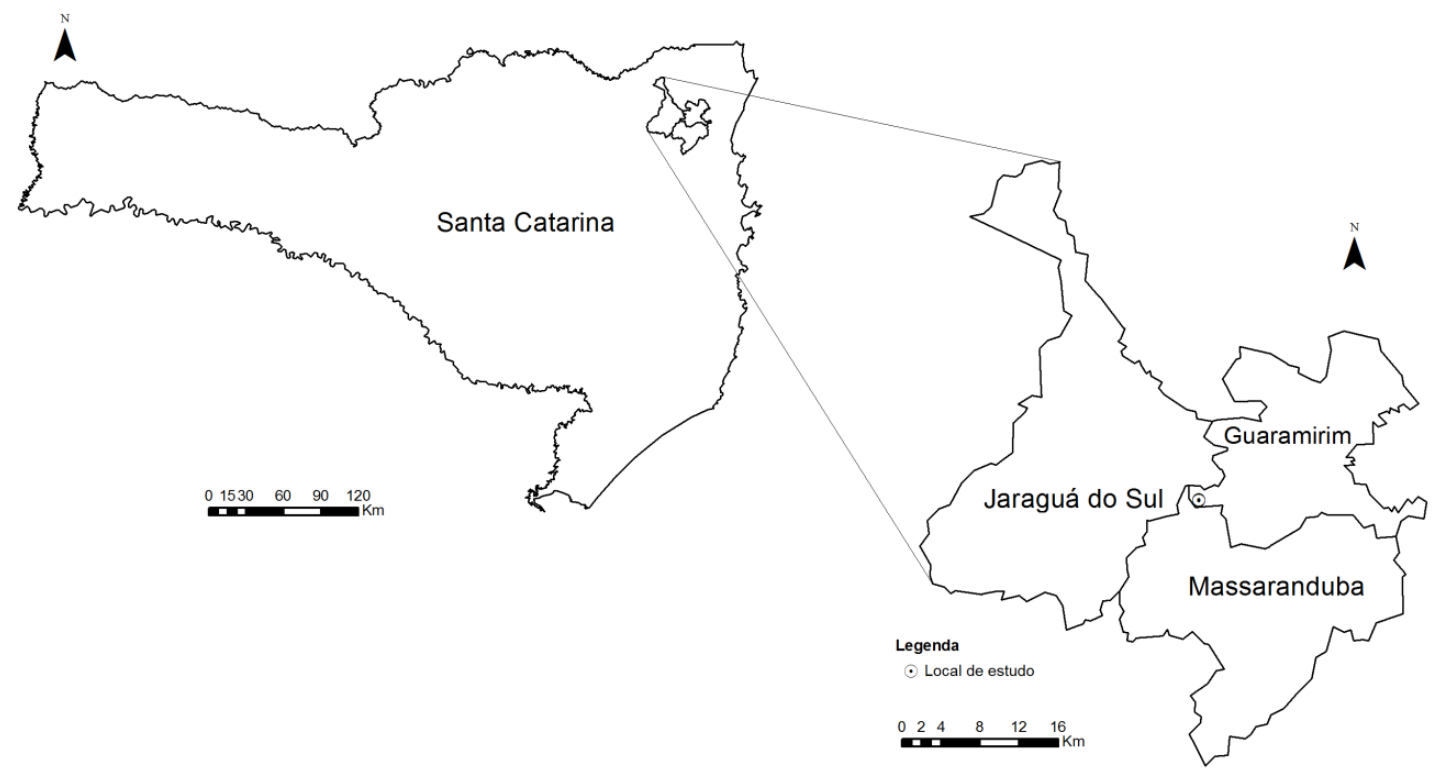

Figura 1. Localização da propriedade em estudo, no município de Guaramirim, na região norte do estado de Santa Catarina, Brasil.

Figure 1. Location of study site, in municipality of Guaramirim, north-eastern Santa Catarina state, Brazil.

estrutura da floresta, sendo encontrado em estudos realizados anteriormente (ex.: Siminski et al., 2004, 2011a). Após o abate de cada árvore, utilizou-se uma trena para medir a altura de fuste (Hf), definida como a altura até o início da copa (primeira bifurcação), e a altura total (Ht). Para determinar o volume do fuste de cada árvore, além da Hf, foram definidas secções delimitadas pelas alturas de $0,1 \mathrm{~m}, 0,3 \mathrm{~m}, 0,7 \mathrm{~m}, 1,0 \mathrm{~m}$, $1,3 \mathrm{~m}, 2,0 \mathrm{~m}$ e, subsequentemente, a cada 1,0 m até a extremidade do fuste, sendo essa delimitada por bifurcações. Em cada uma das extremidades das secções, foram medidos o maior e o menor diâmetro. A partir da primeira bifurcação da árvore, foram medidos o maior e o menor diâmetro de cada galho, a cada 1,0 m, até o diâmetro limite de $5 \mathrm{~cm}$. A cada ponto de medição do diâmetro do tronco e dos galhos, efetuou-se uma medida da espessura de casca simples (Ec) com um paquímetro digital. De todas as árvores foram coletados materiais vegetativos ou férteis, caso presentes, para identificar o nível de espécie. A nomenclatura botânica foi atualizada conforme a Reflora (2015).

\subsection{Análise dos dados}

As árvores amostradas foram divididas em classes diamétricas com intervalo de $5 \mathrm{~cm}$, a fim de se observar a distribuição dos dados. Para estimar o volume de cada secção do tronco e dos galhos, utilizou-se o método de cálculo do volume rigoroso de Smalian, conforme descrição de Finger (1992), por ser o mais aplicado em florestas nativas. De posse desses resultados, foram calculados para cada árvore: volume total com casca (VTCC), volume total sem casca (VTSC), volume de fuste com casca (VFCC) e volume de fuste sem casca (VFSC).

Os volumes das árvores individuais foram, então, submetidos à análise de regressão para testar o seu ajuste a seis modelos florestais clássicos de volumetria (Tabela 1), tendo como variáveis preditoras o DAP, a $\mathrm{Hf}$ e a Ht.

Nos modelos logarítmicos, utilizou-se o fator de Meyer para corrigir as estimativas (Urbano, 2007) e também para recalcular os resíduos. Obteve-se o fator de Meyer a partir da seguinte equação (Urbano, 2007):

Fator de Meyer $=\mathrm{e}^{0,5}$. QMresiduo

Em que e $=2,718282$ e QMresíduo é o quadrado médio do resíduo.

Para selecionar a melhor equação, utilizaram-se a análise de variância da regressão e o teste $t$ de Student comparando o valor de cada coeficiente a zero (Soares et al., 2011). Em seguida, foram analisados o coeficiente de determinação ajustado $\left(R_{a j}^{2}\right)$, o erro-padrão da 
Tabela 1. Modelos testados para a estimativa do volume.

Table 1. Tested models of the volume adjustments.

\begin{tabular}{ccc} 
Modelos & Autores & $\begin{array}{c}\text { Modelo matemático } \\
1\end{array}$ \\
2 & HUSCH & $\ln (v)=b 0+b 1^{*} \ln ($ dap $)+\varepsilon$ \\
3 & SPURR & $v=b 0+b 1^{*} d a p^{2 *} h+\varepsilon$ \\
4 & $\begin{array}{c}\text { SCHUMACHER-HALL } \\
\text { (linearizado) }\end{array}$ & $\ln (v)=b o+b 1^{*} \ln ($ dap $)+b 2^{*} \ln (h)+\varepsilon$ \\
5 & MEYER & $v=b 0+b 1^{*} d a p+b 2^{*} d a p^{2}+b 3^{*} d a p^{*} h+b 4^{*} d a p^{2 *} h+\varepsilon$ \\
6 & STOATE & $v=b 0+b 1^{*} d a p^{2}+b 2^{*} d a p^{2 *} h+b 3^{*} h+\varepsilon$ \\
\hline
\end{tabular}

$\ln$ : logaritmo natural; $v$ : volume $\left(\mathrm{m}^{3}\right)$; dap: diâmetro à altura do peito $(\mathrm{cm}) ; h$ : altura $(\mathrm{m}) ; b 0, b 1, b 2, b 3 \mathrm{e} b 4$ : coeficientes dos modelos; $\varepsilon$ : erro aleatório.

estimativa absoluto (Syx) e em porcentagem (Syx[\%]), o critério de informação de Akaike (AIC) e o critério de informação bayesiano (BIC), seguindo o ranking adaptado de Bartoszeck (2000). Na etapa seguinte, realizou-se análise gráfica dos resíduos em forma porcentual (Silva \& Paula, 1979; Soares et al., 2011).

$\mathrm{O} R_{a j}^{2}$ possibilita mensurar o grau de ajuste ou capacidade de explicação de um modelo, por meio da medida da proporção total da variação em relação à média (Schneider et al., 2009). Em razão de os modelos testados apresentarem números diferentes de coeficientes, é necessário ajustar o $R^{2}$ para que as comparações sejam adequadas, resultando na seguinte expressão (Neter et al., 1996):

$R_{a j}^{2}=1-(n-1 / n-p-1) *($ SQresíduo/SQtotal $)$

Em que $R_{a j}^{2}$ é o coeficiente de determinação ajustado, $n$, o número de observações, $p$, o número de variáveis independentes do respectivo modelo, SQresíduo, a soma de quadrados do resíduo e SQtotal, a soma de quadrados total.

O erro-padrão da estimativa (Syx) indica o erro médio associado ao uso da equação, podendo ser obtido da seguinte maneira (Soares et al., 2011):

Syx $= \pm \sqrt{ }$ QMresíduo

Em que QMresíduo é o quadrado médio do resíduo.

$\mathrm{Na}$ forma porcentual (Syx(\%)), o erro-padrão da estimativa foi obtido pela seguinte equação:

$\operatorname{Syx}(\%)=(\operatorname{Syx} / \ddot{\mathrm{Y}})^{\star} 100$

Em que Syx é o erro-padrão da estimativa e Ÿ, o valor médio da variável dependente.

AIC demonstra a distância geral relativa entre o modelo gerado e o modelo real, sendo, portanto, melhor quanto menor for seu valor, o qual foi obtido com a seguinte equação (Vismara, 2009):

$A I C=-2 \ln (m v)+2 p$

Em que $m v$ é o valor de máxima verossimilhança apresentada pelo modelo e $p$, o número de parâmetros do modelo.

$B I C$ foi desenvolvido de modo a complementar as deficiências do AIC, sendo, de mesma forma, melhor quanto menor for o seu valor (Schwarz, 1978), o qual foi definido como (Carvalho, 2013):

$B I C=-2 \ln (m v)+\ln (n)^{*} p$

Em que $m v$ é o valor de máxima verossimilhança apresentada pelo modelo, $p$, o número de parâmetros do modelo e $n$, o número de observações.

A análise gráfica dos resíduos é muito relevante, pois possibilita identificar problemas como a heterocedasticidade de variâncias mesmo quando a equação demonstra ser precisa (Soares et al., 2011). Além disso, permite detectar tendências resultantes do uso da equação. Os resíduos foram calculados de forma porcentual, de acordo com a seguinte equação:

$\operatorname{Res}(\%)=\frac{\text { Vest }- \text { Vobs }}{\text { Vobs }} * 100$

Em que Res(\%) é o resíduo porcentual, Vest, o valor estimado e Vobs, o valor observado.

O fator de forma reduz o volume do cilindro formado pela área basal e altura para o volume verdadeiro representado pelo sólido de revolução, podendo ser calculado por diversos métodos (Finger, 1992). De acordo com Silva \& Paula (1979), o fator de forma normal $\left(f_{1,30}\right)$ pode ser obtido pela relação entre o volume rigoroso da árvore e o volume do cilindro 
obtido a partir da área basal da árvore a 1,30 $\mathrm{m}$ do solo e da altura do fuste, resumindo-se na seguinte equação (Soares et al., 2011):

$f_{1,30}=\frac{V_{\text {rigoroso }}}{V_{\text {cilindro }}}$

Em que $V_{\text {rigoroso }}$ é o volume obtido a partir da cubagem rigorosa e $V_{\text {cilindro }}$ o volume calculado a partir da área basal a $1,30 \mathrm{~m}$ do solo e da Hf.

$\mathrm{O}$ volume de cada árvore estimado pelo fator de forma foi comparado ao volume real obtido pela cubagem e também ao volume estimado pelo uso da equação de volume correspondente (adaptado de Jorge, 1982; Souza \& Jesus, 1991; Scolforo et al., 1994). Essa comparação, feita por meio de análise de variância de fator único, é útil para testar a eficiência do uso do fator de forma.

A determinação da espessura de casca foi realizada nas mesmas posições determinadas para a cubagem rigorosa, por meio da diferença entre as medições de diâmetro com casca e a dupla espessura da casca. $O$ fator de casca médio $(K)$ pode ser obtido para uma série de dados a partir da seguinte equação (Husch et al., 1982):

$K=\frac{\sum d s c}{\sum d c c}$

Em que $d_{s c}$ é o diâmetro sem casca e $d_{c c}$ o diâmetro com casca.
A partir das derivações dessa equação, é possível obter diversas informações, como volume sem casca, volume de casca, espessura de casca, entre outras muito úteis ao manejo florestal. De forma similar à análise realizada em relação ao fator de forma, utilizou-se análise de variância de fator único para testar se existem diferenças significativas entre o volume real de cada árvore e o volume estimado pelo fator de casca e pelo modelo correspondente (adaptado de Jorge, 1982; Souza \& Jesus, 1991; Scolforo et al., 1994).

Mais detalhes sobre o histórico da área de estudo, metodologia de coleta e análise de dados, assim como informações na íntegra de todas as equações geradas, estão descritos em Correia (2015).

\section{RESULTADOS E DISCUSSÃO}

Foram amostradas 187 árvores, pertencentes a 40 espécies, detalhadas na Tabela 2, com seus respectivos diâmetros médios.

A distribuição nas classes diamétricas (Figura 2) se aproxima da forma de J-invertido, típica de florestas secundárias da região, segundo Siminski et al. (2004, 2011a). As estatísticas descritivas (Tabela 3) apresentam grande variabilidade, principalmente dos volumes, refletindo uma característica de florestas nativas.

Tabela 2. Número de árvores amostradas por espécie $(\mathrm{Na})$ com os valores de DAP médio.

Table 2. Number of trees sampled by specie $(\mathrm{Na})$, with their average $\mathrm{DBH}$.

\begin{tabular}{lccc}
\multicolumn{1}{c}{ Espécie } & Nome popular & Na & DAP \\
Hyeronima alchorneoides Allemão & Licurana ou Urucurana & 35 & 25,7 \\
Miconia cinnamomifolia (DC.) Naudin & Jacatirão & 19 & 26,3 \\
Alchornea triplinervia (Spreng.) Müll.Arg. & Tanheiro & 12 & 21,6 \\
Allophylus petiolulatus Radlk. & Chal-chal & 12 & 9,3 \\
Annona neosericea H. Rainer & Cortiça & 12 & 15,4 \\
Clusia criuva Cambess. & Figueirinha & 7 & 19,0 \\
Guapira opposita (Vell.) Reitz & Maria-mole & 7 & 16,5 \\
Matayba intermedia Radlk. & Camboatá & 7 & 18,3 \\
Cupania oblongifolia Mart. & Miguel-pintado & 6 & 14,5 \\
Nectandra membranacea (Sw.) Griseb. & Canela-branca & 6 & 29,0 \\
Virola bicuhyba (Schott ex Spreng.) Warb. & Bocuba ou virola & 6 & 26,2 \\
Nectandra oppositifolia Nees & Canela-garuva & 5 & 29,7 \\
Casearia decandra Jacq. & Cafezeiro & 4 & 10,8 \\
Citharexylum myrianthum Cham. & Tucaneira & 4 & 29,7 \\
Miconia cabucu Hoehne & Pau-ferro & 4 & 15,2 \\
Myrcia spectabilis DC. & Guamirim & 4 & 6,9 \\
Demais espécies (24) & & 37 & 19,2 \\
Total & - & $\mathbf{1 8 7}$ & $\mathbf{2 0 , 7}$ \\
\hline
\end{tabular}


A dispersão dos volumes em relação ao DAP (Figura 3) apresenta tendência exponencial tanto para valores com casca quanto sem casca.

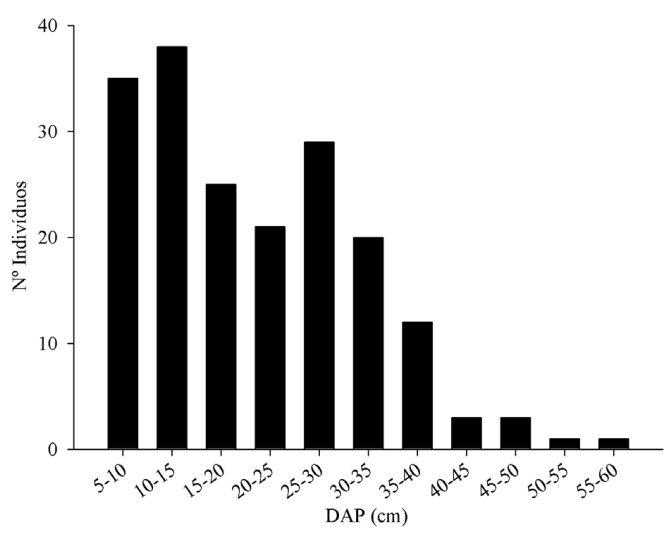

Figura 2. Distribuição do número de árvores amostradas por classe diamétrica.

Figure 2. Distribution of the number of sampled trees by diameter class.
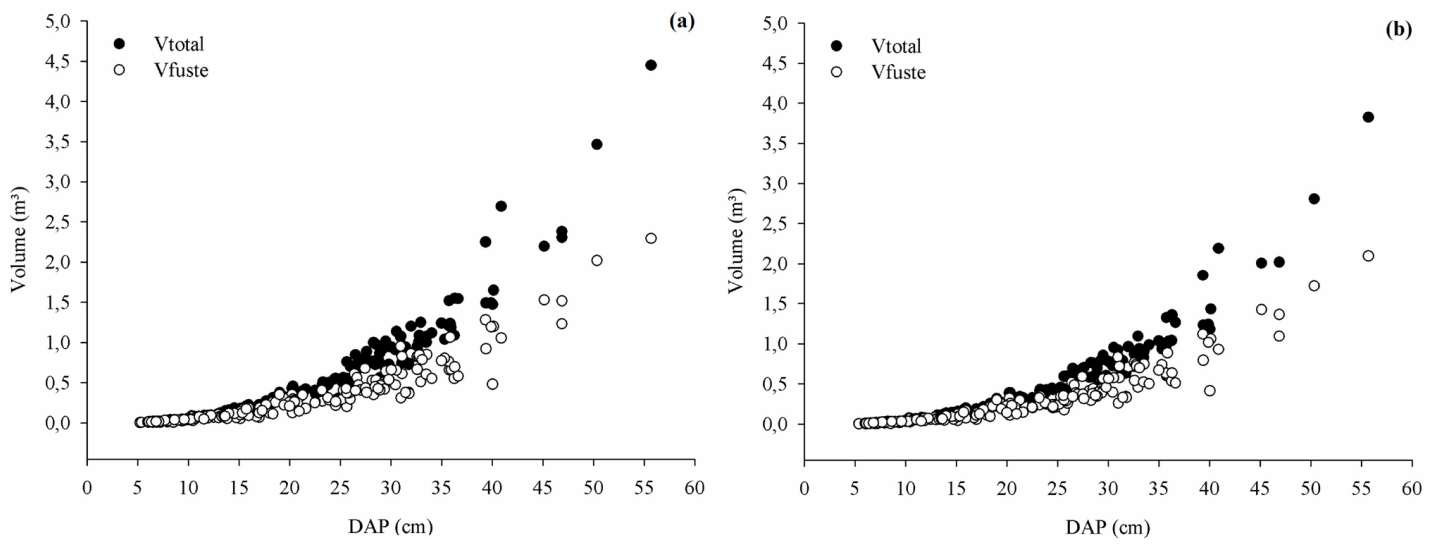

Figura 3. Dispersão dos volumes com casca (a) e sem casca (b) em função do diâmetro à altura do peito (DAP). Figure 3. Dispersion of volume with bark (a) and without bark (b) as a function of the Diameter at Breast Height (DBH).

Tabela 3. Estatísticas descritivas para as árvores cubadas.

Table 3. Descriptive statistics for the scaled trees.

\begin{tabular}{lcccccccc}
\multicolumn{1}{c}{ Estatística } & $\begin{array}{c}\text { DAP } \\
(\mathbf{c m})\end{array}$ & $\begin{array}{c}\text { Ht } \\
(\mathbf{m})\end{array}$ & $\begin{array}{c}\text { Hf } \\
(\mathbf{m})\end{array}$ & $\begin{array}{c}\text { Ec } \\
(\mathbf{m m})\end{array}$ & $\begin{array}{c}\text { VFCC } \\
\left(\mathbf{m}^{3}\right)\end{array}$ & $\begin{array}{c}\text { VTCC } \\
\left(\mathbf{m}^{3}\right)\end{array}$ & $\begin{array}{c}\text { VFSC } \\
\left(\mathbf{m}^{3}\right)\end{array}$ & $\begin{array}{c}\text { VTSC } \\
\left(\mathbf{m}^{3}\right)\end{array}$ \\
\hline Mediana & 19,1 & 16,1 & 8,0 & 6,00 & 0,2160 & 0,2795 & 0,1907 & 0,2409 \\
Média & 20,7 & 15,9 & 8,7 & 6,49 & 0,3236 & 0,5142 & 0,2834 & 0,4352 \\
\hline Variância & 115,8 & 26,3 & 14,6 & 9,41 & 0,1369 & 0,4099 & 0,1077 & 0,2941 \\
Desvio-padrão & 10,8 & 5,1 & 3,8 & 3,07 & 0,3700 & 0,6402 & 0,3281 & 0,5423 \\
$\begin{array}{l}\text { Coeficiente de } \\
\text { variação (\%) }\end{array}$ & 52,2 & 32,1 & 43,7 & 47,3 & 114,3 & 124,5 & 115,8 & 124,6 \\
\hline
\end{tabular}

\subsection{Modelos de volume total}

Todos os modelos testados apresentaram alto $R_{a j}^{2}$ e baixos valores de $\operatorname{Syx}(\%)$ e ambos os valores dessas estatísticas em todos os modelos foram semelhantes entre si (Tabela 4). Segundo esses critérios de avaliação, tanto os modelos com casca quanto os sem casca seriam adequados para estimar os volumes correspondentes. Diferenças mais evidentes foram obtidas nas avaliações dos modelos usando como critérios AIC e BIC, que revelam superioridade dos modelos 1 e 3 . Com base no ranking dessas estatísticas, tais modelos foram os melhores para VTCC e o modelo 1 foi o melhor para VTSC, porém muito próximo ao modelo 3. Como o modelo 1 se refere ao de Husch e o modelo 3, ao de Schumacher-Hall (linearizado), pode-se concluir previamente que houve melhor ajuste dos dados aos modelos logarítmicos (variáveis preditoras e respostas transformadas). Tanto para o volume com casca quanto para o sem casca, os modelos 1 e 3 apresentaram todos os coeficientes significativos (Tabela 5) e a escolha 
Tabela 4. Critérios de seleção dos modelos de volume avaliados.

Table 4. Selection criteria of the volume models evaluated.

\begin{tabular}{|c|c|c|c|c|c|c|c|c|c|c|c|}
\hline Volume & Modelo & Syx & $\begin{array}{l}\text { Syx } \\
(\%)\end{array}$ & $\mathbf{C}^{*}$ & $\boldsymbol{R}^{2}{ }_{a j .}$ & $\mathbf{C}^{*}$ & $A I C$ & $\mathbf{C}^{*}$ & BIC & $\mathbf{C}^{*}$ & Rank. \\
\hline \multirow{6}{*}{$\begin{array}{l}\text { Total com } \\
\text { casca }\end{array}$} & 1 & 0,1518 & 29,5 & 6 & 0,97 & 2 & 16,79 & 1 & 26,49 & 1 & 10 \\
\hline & 2 & 0,1406 & 27,3 & 4 & 0,95 & 4 & $-198,35$ & 3 & $-188,66$ & 3 & 14 \\
\hline & 3 & 0,1483 & 28,8 & 5 & 0,98 & 1 & $-41,24$ & 2 & $-28,31$ & 2 & 10 \\
\hline & 4 & 0,1276 & 24,8 & 2 & 0,96 & 3 & $-232,52$ & 5 & $-213,13$ & 5 & 15 \\
\hline & 5 & 0,1291 & 25,1 & 3 & 0,96 & 3 & $-229,11$ & 4 & $-212,95$ & 4 & 14 \\
\hline & 6 & 0,1247 & 24,3 & 1 & 0,96 & 3 & $-240,89$ & 6 & $-221,50$ & 6 & 16 \\
\hline \multirow{6}{*}{$\begin{array}{l}\text { Total sem } \\
\text { casca }\end{array}$} & 1 & 0,1141 & 26,2 & 6 & 0,98 & 1 & 2,38 & 1 & 12,05 & 1 & 9 \\
\hline & 2 & 0,1102 & 25,3 & 4 & 0,96 & 3 & $-287,10$ & 3 & $-277,44$ & 3 & 13 \\
\hline & 3 & 0,1118 & 25,7 & 5 & 0,98 & 1 & $-57,04$ & 2 & $-44,16$ & 2 & 10 \\
\hline & 4 & 0,0990 & 22,7 & 2 & 0,97 & 2 & $-323,76$ & 5 & $-304,44$ & 5 & 14 \\
\hline & 5 & 0,1008 & 23,2 & 3 & 0,97 & 2 & $-318,21$ & 4 & $-302,10$ & 4 & 13 \\
\hline & 6 & 0,0967 & 22,2 & 1 & 0,97 & 2 & $-332,61$ & 6 & $-313,29$ & 6 & 15 \\
\hline \multirow{6}{*}{$\begin{array}{l}\text { Fuste com } \\
\text { casca }\end{array}$} & 1 & 0,1142 & 35,3 & 6 & 0,95 & 4 & 105,62 & 1 & 115,31 & 1 & 12 \\
\hline & 2 & 0,0768 & 23,7 & 5 & 0,96 & 3 & $-425,19$ & 3 & $-415,50$ & 3 & 14 \\
\hline & 3 & 0,0507 & 15,7 & 2 & 0,99 & 1 & $-196,64$ & 2 & $-183,72$ & 2 & 7 \\
\hline & 4 & 0,0527 & 16,3 & 3 & 0,98 & 2 & $-563,02$ & 5 & $-543,64$ & 5 & 15 \\
\hline & 5 & 0,0543 & 16,8 & 4 & 0,98 & 2 & $-552,79$ & 4 & $-536,64$ & 4 & 14 \\
\hline & 6 & 0,0483 & 14,9 & 1 & 0,98 & 2 & $-595,48$ & 6 & $-576,09$ & 6 & 15 \\
\hline \multirow{6}{*}{$\begin{array}{l}\text { Fuste sem } \\
\text { casca }\end{array}$} & 1 & 0,0959 & 33,8 & 6 & 0,95 & 3 & 95,75 & 1 & 105,41 & 1 & 11 \\
\hline & 2 & 0,0728 & 25,7 & 5 & 0,95 & 3 & $-440,22$ & 3 & $-430,56$ & 3 & 14 \\
\hline & 3 & 0,0468 & 16,5 & 2 & 0,99 & 1 & $-166,95$ & 2 & $-154,06$ & 2 & 7 \\
\hline & 4 & 0,0489 & 17,3 & 3 & 0,98 & 2 & $-584,78$ & 5 & $-565,45$ & 5 & 15 \\
\hline & 5 & 0,0516 & 18,2 & 4 & 0,98 & 2 & $-565,55$ & 4 & $-549,44$ & 4 & 14 \\
\hline & 6 & 0,0445 & 15,7 & 1 & 0,98 & 2 & $-619,38$ & 6 & $-600,06$ & 6 & 15 \\
\hline
\end{tabular}

${ }^{*}$ C: classificação do modelo no critério avaliado. Em negrito, os modelos de destaque.

do melhor entre eles se baseou, então, na análise dos resíduos do modelo ajustado (Figura 4).

Tanto no modelo 1 quanto no modelo 3 , a maioria dos resíduos se apresentou bem distribuída em relação às classes diamétricas, estando concentrados entre - 40\% e 40\% (Figura 4a, b). Há tendência de superestimar o volume nas classes de diâmetro inferiores (DAP $<30 \mathrm{~cm}$ ) em ambos os modelos, com resíduos alcançando valores próximos a $120 \%$. No modelo 3 (Figura $4 \mathrm{~b}$ ), há menos ocorrências de superestimação do volume nas classes de menor DAP, sendo, por isso, superior ao modelo 1 para predizer valores de VTCC, resultando na equação em destaque na Tabela 5.

Comportamento similar foi observado para os modelos de estimativa do volume total sem casca (Figura 4c, d) e, novamente, o modelo 3 apresentou menor número de casos de superestimativa do volume, sendo, assim, o recomendado (em destaque na Tabela 5).
Em um dos poucos estudos que relatam descrições de modelos de volume total com casca (até o limite de $5 \mathrm{~cm}$ de diâmetro, incluindo galhos) para espécies nativas da FOD de SP, Nunes (2013) obteve também um bom ajuste, com $R_{a j}^{2}=0,97$, valor próximo ao verificado no presente estudo. No caso de modelos de VTSC, não foram encontrados estudos similares que possibilitassem comparações.

\subsection{Modelos de volume de fuste}

Em relação ao volume do fuste com casca, também houve bom ajuste dos dados aos modelos testados, com baixos valores de $S y x(\%)$ e altos valores de $R_{a j}^{2}$ (Tabela 4). Novamente, destacou-se o modelo 3, com bons valores de AIC e BIC. Os resultados para o volume do fuste sem casca foram similares, com bons ajustes, particularmente para o modelo 3 . Todos os coeficientes foram significativos para o modelo $3 \mathrm{em}$ ambos os volumes (Tabela 5). Os resíduos do modelo 3 
Tabela 5. Coeficientes dos modelos de volume avaliados.

Table 5. Coefficients for the volume models evaluated.

\begin{tabular}{|c|c|c|c|c|c|c|}
\hline Volume & Modelo & bo & b1 & b2 & b3 & b4 \\
\hline \multirow{6}{*}{$\begin{array}{l}\text { Total com } \\
\text { casca }\end{array}$} & 1 & $-9,353161$ & 2,710278 & - & - & - \\
\hline & 2 & $0,010669^{\mathrm{NS}}$ & 0,000047 & - & - & - \\
\hline & 3 & $-10,045586$ & 2,349493 & 0,640598 & - & - \\
\hline & 4 & $0,020121^{\mathrm{NS}}$ & $-0,002416^{\mathrm{NS}}$ & 0,000696 & $-0,000450^{\mathrm{NS}}$ & 0,000031 \\
\hline & 5 & $0,049422^{\mathrm{NS}}$ & 0,000497 & 0,00003 & $-0,008108$ & - \\
\hline & 6 & $-0,045741^{\mathrm{NS}}$ & $0,000191^{\mathrm{NS}}$ & 0,000068 & $-0,000064$ & $0,000680^{\mathrm{NS}}$ \\
\hline \multirow{6}{*}{$\begin{array}{c}\text { Total sem } \\
\text { casca }\end{array}$} & 1 & $-9,495131$ & 2,698192 & - & - & - \\
\hline & 2 & $0,003505^{\mathrm{NS}}$ & 0,00004 & - & - & - \\
\hline & 3 & $-10,206982$ & 2,348857 & 0,634736 & - & - \\
\hline & 4 & $0,033057^{\mathrm{NS}}$ & $-0,004325^{\mathrm{NS}}$ & 0,000613 & $-0,000350^{\mathrm{NS}}$ & 0,000027 \\
\hline & 5 & $0,049941^{\mathrm{NS}}$ & 0,000382 & 0,000027 & $-0,007538$ & - \\
\hline & 6 & $-0,038219^{\mathrm{NS}}$ & $0,000118^{\mathrm{NS}}$ & 0,00006 & $-0,000055$ & 0,000576 \\
\hline \multirow{6}{*}{$\begin{array}{l}\text { Fuste com } \\
\text { casca }\end{array}$} & 1 & $-8,839886$ & 2,419584 & - & - & - \\
\hline & 2 & 0,01876 & 0,000056 & - & - & - \\
\hline & 3 & $-9,285656$ & 2,100113 & 0,665269 & - & - \\
\hline & 4 & $0,043718^{\mathrm{NS}}$ & $-0,008695$ & 0,000467 & $0,000304^{\mathrm{NS}}$ & 0,000027 \\
\hline & 5 & $-0,010852^{\mathrm{NS}}$ & 0,000252 & 0,000037 & $-0,000647^{\text {NS }}$ & - \\
\hline & 6 & $-0,018057$ & 0,000118 & 0,000065 & $-0,000075$ & 0,000947 \\
\hline \multirow{6}{*}{$\begin{array}{l}\text { Fuste sem } \\
\text { casca }\end{array}$} & 1 & $-9,029642$ & 2,431297 & - & - & - \\
\hline & 2 & $0,011545^{\mathrm{NS}}$ & 0,000049 & - & - & - \\
\hline & 3 & $-9,504432$ & 2,140098 & 0,637631 & - & - \\
\hline & 4 & 0,053711 & $-0,009812$ & 0,000471 & $0,000259^{\mathrm{NS}}$ & 0,000022 \\
\hline & 5 & $-0,006918^{\mathrm{NS}}$ & 0,000226 & 0,000033 & $-0,001835^{\mathrm{NS}}$ & - \\
\hline & 6 & $-0,019814$ & 0,000089 & 0,000061 & $-0,000077$ & 0,000929 \\
\hline
\end{tabular}

${ }^{\text {Ns }}$ Coeficiente não significativo pelo teste $\mathrm{t}$ a um nível de probabilidade de 5\%. b0, b1, b2, b3 e b4 se referem aos coeficientes dos modelos. Em negrito, os modelos selecionados.
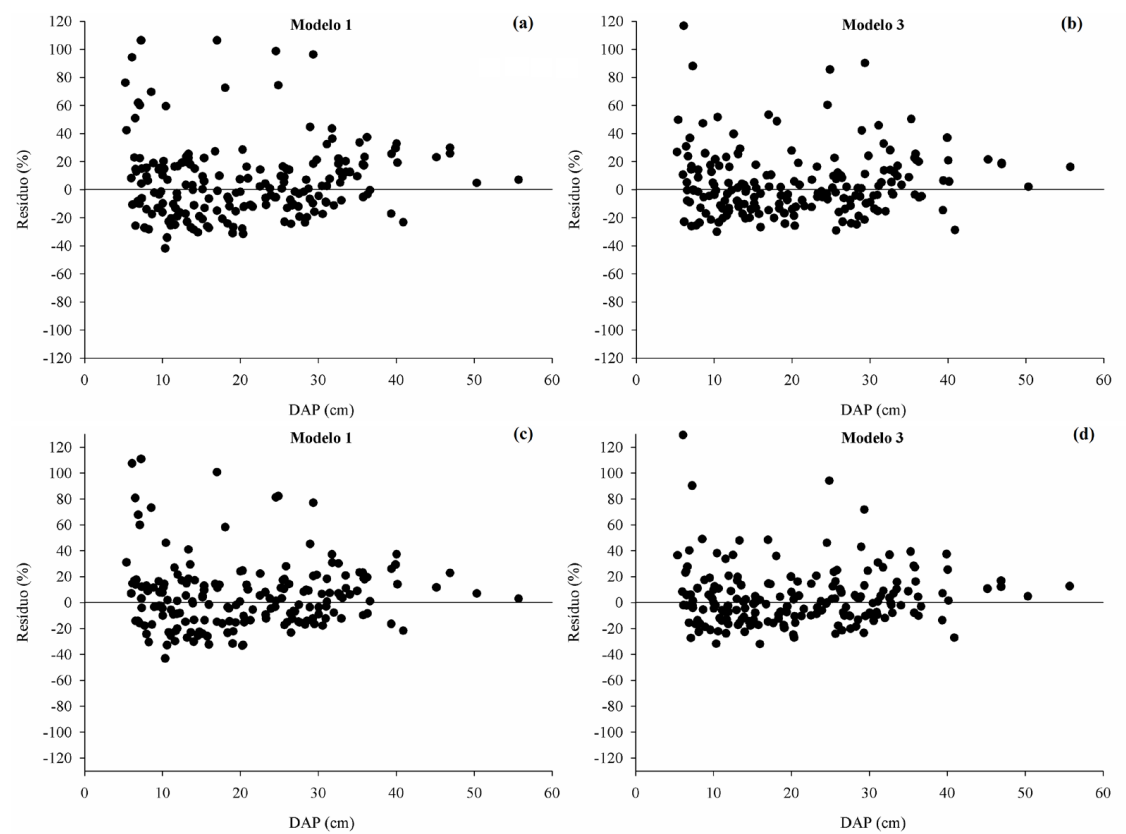

Figura 4. Resíduos porcentuais dos modelo 1 (a) e 3 (b) para estimar o volume total com casca e dos modelos 1 (c) e 3 (d) para estimar o volume total sem casca em função do DAP.

Figure 4. Percent residuals of Model 1 (a) and Model 3 (b) for the estimation of the total volume with bark, and of Model 1 (c) and Model 3 (d) for the estimation of the total volume without bark, as a function of the DBH. 
para o volume de fuste com casca se mostraram bem distribuídos, com valores entre -20\% e 40\% (Figura 5a), resultando, portanto, na recomendação desse modelo para a estimativa proposta (Tabela 5).

Os resíduos do modelo 3 ajustado para estimativa do volume do fuste sem casca apresentaram comportamento similar (Figura 5b), sendo, novamente, a equação recomendada para esse propósito (Tabela 5).

Em pesquisas para FOD do ES, Jorge (1982) também obteve o modelo de Schumacher-Hall (linearizado) entre os de melhor ajuste para volume comercial com casca, com valores de $R_{a j}^{2}$ acima de 0,90 , $\operatorname{Syx}(\%)=12 \%$, inferior ao encontrado neste estudo, $\mathrm{e}$ com resíduos distribuídos de forma próxima, entre -30 e 30\%. Chichorro et al. (2003) ajustaram o modelo de Schumacher-Hall (linearizado) para esse mesmo tipo florestal do ES e obtiveram $R_{a j}^{2}=0,98$. De forma similar, em estudos de FOD do ES, Souza \& Jesus (1991) obtiveram o modelo de Schumacher-Hall (linearizado) como o mais adequado, embora os autores estivessem trabalhando com modelos de espécies individuais. Vibrans et al. (2012b) também encontraram o modelo de Schumacher-Hall (linearizado e adaptado) como o mais adequado para estimativas de VFCC para a FOD de SC, $\operatorname{com} R_{a j}^{2}=0,93$ e Syx $(\%)=2,19 \%$. Da mesma forma, Silveira (2008) obteve bons ajustes para modelos de volume comercial com casca em FOD de SC, com $R_{a j}^{2}=0,97$ e $\operatorname{Syx}(\%)$ de aproximadamente $13 \%$, porém com modelo não logarítmico (Stoate).
Dessa forma, os resultados obtidos neste estudo corroboram os poucos relatos encontrados na literatura, sendo um indicativo da qualidade das equações ante a escassez de literatura para comparações. Salienta-se, ainda, a precisão dos dados coletados neste estudo, cujas fontes de erros foram reduzidas por conta de serem utilizadas árvores abatidas.

\subsection{Fator de forma}

O fator de forma é uma informação útil em avaliações expeditas de volume aproveitável de árvores para produção de madeira serrada, e não havia estudo para essa variável para florestas secundárias da FOD. O cálculo do fator forma normal médio $\left(f_{1,3}\right)$ resultou no valor de 0,8027 . Outros estudos relatam um fator de forma de 0,77 para a FOM do RS (Borsoi, 2005) e de 0,827 para a FES de Minas Gerais (Scolforo et al., 1994). Outros estudos foram feitos para espécies individualmente: Schuch et al. (2008), em SC, que estimaram fator de forma de 0,92 para M. cinnamomifolia; Souza \& Jesus (1991), que relataram fator de forma, respectivamente, de 0,6293, 0,6137, 0,7027 e 0,6792 para Cordia trichotoma, Cariniana legalis, Bowdichia virgilioides e Joannesia princeps, no ES, e Durlo \& Denardi (2009), que estimaram fator de forma de 0,84 para Cabralea canjerana na FED do RS. Mesmo com diferenças na metodologia utilizada, tais resultados denotam, como esperado para espécies nativas, grande variabilidade dos valores de fatores de forma.
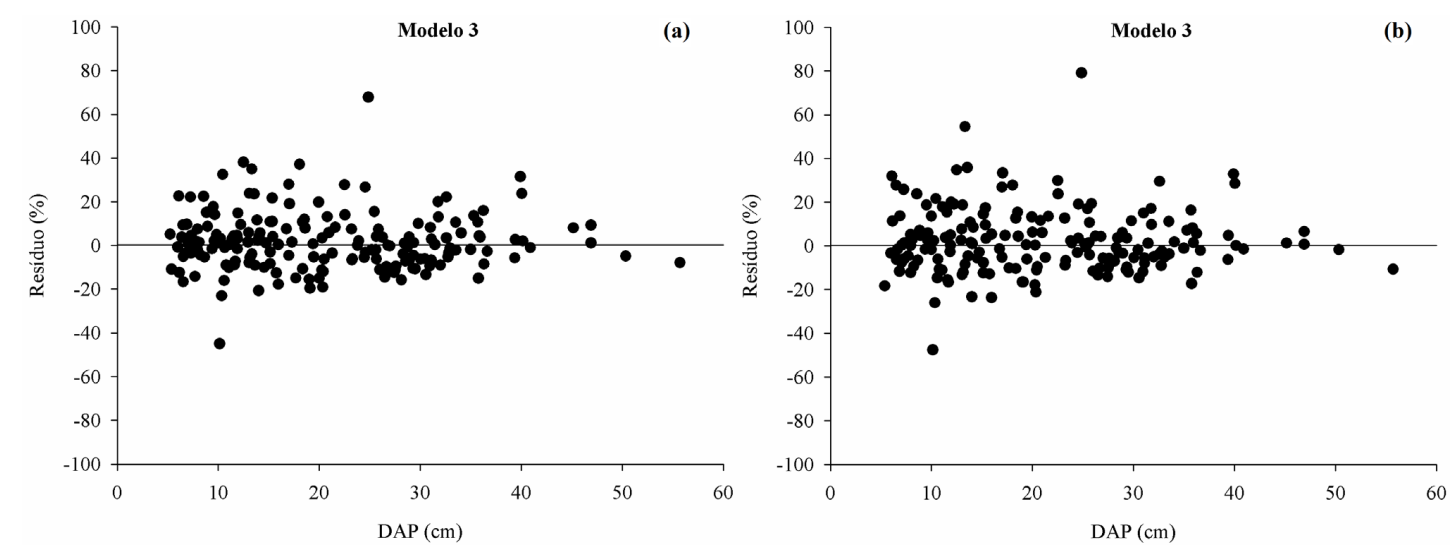

Figura 5. Resíduos porcentuais do modelo 3 para estimar os volumes de fuste com casca (a) e sem casca (b) em função do DAP.

Figure 5. Percent residuals of Model 3 for the estimation of the stem volume with bark (a) and without bark (b), depending on the $\mathrm{DBH}$. 

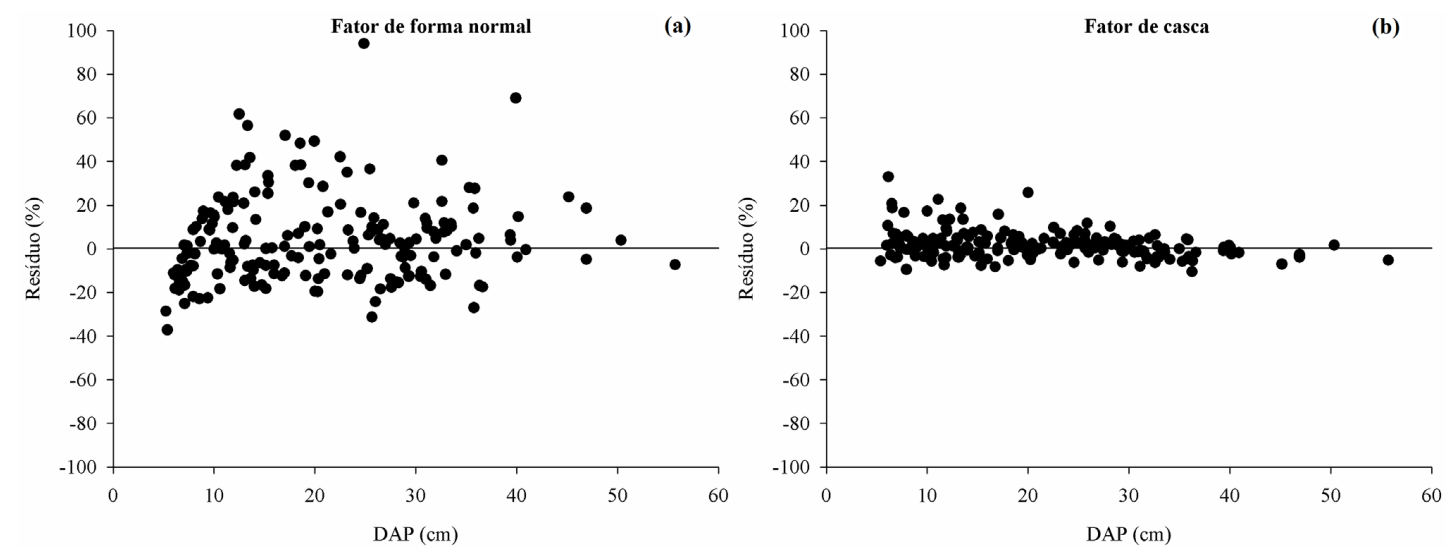

Figura 6. Resíduos porcentuais da medição do volume obtido por meio de cubagem rigorosa em relação à estimativa do volume de fuste com casca obtido a partir do fator de forma normal (a) e do volume de fuste sem casca obtido a partir do fator de casca (b), em função do DAP.

Figure 6. Percent residuals of the volume measured and the estimated stem volume with bark obtained with the normal form factor (a), and the stem volume without bark obtained with the bark factor (b), as a function of the $\mathrm{DBH}$.

Por meio de análise de variância, compararam-se o volume com casca do fuste de cada árvore estimado pelo modelo selecionado (Tabela 5 ), as estimativas obtidas utilizando-se o fator de forma $\left(f_{1,3}\right)$ e os valores da cubagem rigorosa, tendo se constatado que não houve diferença estatística entre os valores $\left(\mathrm{F}_{\text {calc }}=0,17\right.$; $p=0,85$ ). Souza \& Jesus (1991) e Scolforo et al. (1994) também realizaram teste semelhante e igualmente não verificaram diferenças significativas entre estimativas de volume. Além disso, foram plotados os resíduos porcentuais da estimativa do volume de fuste com casca obtido a partir do fator de forma normal e o volume correspondente obtido através da cubagem rigorosa, em função do DAP (Figura 6a). A comparação desses resíduos com os resíduos das estimativas de volume do fuste com casca do modelo selecionado (Figura 5a) evidencia que o fator de forma apresentou desempenho inferior, com maior número de volumes superestimados.

Os resultados aqui apresentados sugerem que o fator de forma pode ser usado para estimativas expeditas de volume do fuste. Entretanto, quando estimativas mais precisas são necessárias, recomenda-se a aplicação das equações de volume.

\subsection{Fator de casca}

O fator de casca $(K)$ obtido em relação ao volume de fuste foi de 0,9301 . Os poucos relatos dessa variável para espécies nativas da Mata Atlântica são de
Schuch et al. (2008), para M. cinnamomifolia (0,91), e de Borsoi (2005), para a FOM do RS $(0,9456)$. Apesar de escassos, os valores comparativos estão próximos, salientando-se que o mais adequado seria a comparação entre formações florestais similares e entre espécies, sempre que possível.

A análise de variância revelou que não há diferença estatística significativa $\left(\mathrm{F}_{\text {calc }}=0,01 ; p=0,99\right)$ entre o volume do fuste sem casca da cubagem rigorosa, o volume estimado pelo modelo selecionado (Tabela 5) e o volume estimado pelo uso de $K$ (relacionando o VFCC real e o $K$ ). A maioria dos desvios dos volumes estimados com uso do fator de casca, em relação aos volumes obtidos na cubagem rigorosa, oscila entre $-10 \%$ e $20 \%$ (Figura 6b), amplitude menor que os resíduos gerados pelo modelo selecionado para estimar o volume do fuste sem casca (Figura 5b). Portanto, apesar de o fator de casca apresentar tendência em superestimar os volumes nas classes de diâmetro abaixo de $20 \mathrm{~cm}$, com pontos ultrapassando $30 \%$, seu uso é recomendado.

\section{CONCLUSÕES}

Todos os modelos testados apresentaram bom ajuste para as estimativas de volumes total e de fuste, tanto com casca quanto sem casca, com destaque para o modelo de Schumacher-Hall (linearizado), sendo esse, portanto, recomendado para estimar o volume 
de florestas secundárias da FOD da região sul da Mata Atlântica.

As diferenças entre as estimativas obtidas por meio do uso dos modelos e as obtidas pelo uso do fator de forma e do fator de casca não foram diferentes estatisticamente. Entretanto, a análise dos resíduos revelou que a equação de volume selecionada apresentou desempenho superior ao fator de forma, sendo, portanto, preferível para estimar o volume do fuste com casca. No caso da estimativa do volume do fuste sem casca, o fator de casca apresentou menor amplitude dos resíduos quando comparado ao modelo selecionado, sendo por isso preferível.

\section{AGRADECIMENTOS}

Este estudo foi realizado no âmbito do termo de cooperação técnico-científica ( $n^{\circ}$ 10/2003) entre Universidade Federal de Santa Catarina (UFSC) e Fundação do Meio Ambiente (FATMA), teve financiamento da Fundação de Apoio à Pesquisa Científica e Tecnológica do Estado de Santa Catarina (FAPESC) e Conselho Nacional de Desenvolvimento Científico e Tecnológico (CNPq) (5287/2011-6). O primeiro e terceiro autores foram bolsistas de mestrado do $\mathrm{CNPq}$ e o segundo autor, bolsista de produtividade do CNPq. Os autores agradecem a dois revisores anônimos pelas contribuições que melhoraram significativamente o artigo.

\section{STATUS DA SUBMISSÃO}

Recebido: 16 mar., 2016

Aceito: 7 set., 2016

\section{AUTOR(ES) PARA CORRESPONDÊNCIA}

\section{Jean Correia}

Departamento de Fitotecnia, Universidade Federal de Santa Catarina - UFSC, Rodovia Admar Gonzaga, 1346, Itacorubi, CEP 88040-900, Florianópolis, SC, Brasil e-mail: jeancorreia@florestal.eng.br

\section{REFERENCIAS}

Barrance A, Schreckenberg K, Gordon J. Conservation through use: lessons from the mesoamerican dry forest. London: Overseas Development Institute; 2009.
Bartoszeck A. Evolução da distribuição diamétrica e da relação hipsométrica em função dos fatores idade, sítio e densidade inicial em bracatingais da região metropolitana de Curitiba [dissertação]. Curitiba: Departamento de Engenharia Florestal, Universidade Federal do Paraná; 2000.

Borsoi, GA. Subsídios para o manejo de uma floresta ombrófila mista em estágio avançado de regeneração natural [tese]. Santa Maria: Departamento de Engenharia Florestal, Universidade Federal de Santa Maria; 2005.

Carvalho SDPC. Estimativa volumétrica por modelo misto e tecnologia laser aerotransportado em plantios clonais de Eucalyptus sp. [tese]. Piracicaba: Departamento de Engenharia Florestal, Universidade de São Paulo; 2013.

Chichorro JF, Resende JLP, Leite HG. Equações de volume e de taper para quantificar multiprodutos da madeira em floresta atlântica. Revista Árvore. 2003; 27(6): 799-809. http://dx.doi.org/10.1590/S0100-67622003000600006.

Correia J. Modelos volumétricos para florestas secundárias do litoral de Santa Catarina [dissertação]. Florianópolis: Centro de Ciências Agrárias, Universidade Federal de Santa Catarina; 2015.

Dean W. A ferro e fogo: a história e a devastação da Mata Atlântica brasileira. São Paulo: Companhia das Letras; 1996.

Durlo MA, Denardi L. Morfometria de Cabralea canjerana, em mata secundaria nativa do Rio Grande do Sul. Ciência Florestal 2009; 8(1): 55-66.

Fantini AC, Siminski A. Espécies madeireiras nativas da Região Sul do Brasil. In: Coradin L, Siminski A, Reis A. Espécies nativas da flora brasileira de valor econômico atual ou potencial: plantas para o futuro - Região Sul. Brasília: MMA; 2011.

Finger CAG. Medição de árvores: fundamentos de biometria florestal. Santa Maria: UFSM/CEPEF/FATEC; 1992.

Fundação SOS Mata Atlântica. Atlas dos remanescentes florestais da Mata Atlântica: período 2011-2012. São Paulo; 2013.

Husch B, Miller CI, Beers TW. Forest mensuration. Florida: Krieger Publishing Company; 1982.

Instituto Brasileiro de Geografia e Estatística - IBGE. Manual técnico da vegetação brasileira. Brasília; 2012.

Jorge LAB. Equações de volume comercial com casca em floresta tropical pluvial no norte do Espírito Santo. In: Anais do Congresso Brasileiro sobre Essências Nativas; 1982, Campos do Jordão. São Paulo: Instituto Florestal; 1982. p. 456.

Klein RM. Mapa fitogeográfico do Estado de Santa Catarina. In: Reitz R, editor. Flora ilustrada catarinense. Itajaí: Herbário Barbosa Rodrigues; 1978.

Neter J, Wasserman W, Kutner MH. Applied linear statistical models. Chicago: Irwin; 1996.

Nunes MH. Stem profile modeling in Cerrado and tropical forests formations in Brazil [dissertação]. Piracicaba: 
Departamento de Engenharia Florestal, Universidade de São Paulo; 2013.

Oller DC. Ecologia e manejo de florestas secundárias dominadas por Tibouchina pulchra Cogn [dissertação]. Florianópolis: Centro de Ciências Agrárias, Universidade Federal de Santa Catarina; 2011.

Redford KH, Richter BD. Conservation of biodiversity in a world of use. Conservation Biology 1999; 13(6): 12461256. http://dx.doi.org/10.1046/j.1523-1739.1999.97463.x.

Reflora. Flora do Brasil 2020. Rio de Janeiro; 2015 [citado em 2015 jan. 15]. Avaliable from: http://floradobrasil. jbrj.gov.br/.

Santos K, Sanquetta CR, Eisfield RDL, Watzlawick LF, Ziliotto MAB. Equações volumétricas por classe diamérica para algumas espécies folhosas da Floresta Ombrófila Mista no Paraná, Brasil. RECEN-Revista Ciências Exatas e Naturais. 2009; 8(1): 99-112.

Schneider PR, Schneider P, Souza CD. Análise de regressão aplicada à Engenharia Florestal. Santa Maria: UFSM/ CEPEF; 2009.

Schuch C, Siminski A, Fantini AC. usos e potencial madeireiro do jacatirão-açu (Miconia cinnamomifolia (de candolle) naudin) no litoral de Santa Catarina. Floresta. 2008; 38(4): 735-741. http://dx.doi.org/10.5380/ rf.v38i4.13169.

Schwarz G. Estimating the dimension of a model. Annals of Statistics 1978; 6(2): 461-464. http://dx.doi.org/10.1214/ aos/1176344136.

Scolforo J, Mello JD, Lima CDA. Obtenção de relações quantitativas para estimativa de volume do fuste em floresta estacional semidecídua montana. Revista Cerne. 1994; 1(1): 123-134.

Silva JAA, Paula F No. Princípios básicos de dendrometria. Recife: UFRPE; 1979.

Silveira P. Métodos indiretos de estimativa do conteúdo de biomassa e do estoque de carbono em um fragmento de floresta ombrófila densa [tese]. Curitiba: Departamento de Engenharia Florestal, Universidade Federal do Paraná; 2008.
Siminski A, Fantini AC. A Mata Atlântica cede lugar a outros usos da terra em Santa Catarina, Brasil. Biotemas. 2010; 23(2): 51-59.

Siminski A, Fantini AC, Guries RP, Ruschel A, Reis MS. Secondary forest succession in the Mata Atlantica, Brazil: floristic and phytosociologic trends. ISRN Ecology. 2011a;2011: 1-19.

Siminski A, Santos KLD, Fantini AC, Reis MS. Recursos florestais nativos e a agricultura familiar em Santa Catarina-Brasil. Bonplandia. 2011b; 20(2): 371-389.

Siminski A, Mantovani M, Reis MS, Fantini AC. Sucessão florestal secundária no município de São Pedro de Alcântara, litoral de Santa Catarina: estrutura e diversidade. Ciência Florestal 2004; 14(1): 21-33.

Soares CPB, Paula F No, Souza AL. Dendrometria e inventário florestal. Viçosa: UFV; 2011.

Souza A, Jesus R. Equações de volume comercial e fator de forma para espécies da Mata Atlântica ocorrentes na reserva florestal da Companhia Vale do Rio Doce, Linhares, ES. Revista Árvore. 1991;15(3):257-73.

Urbano E. Quantificação e estimativa aérea da biomassa e do carbono fixado em árvores de bracatingais nativos da região metropolitana de Curitiba [dissertação]. Curitiba: Departamento de Engenharia Florestal, Universidade Federal do Paraná; 2007.

Vibrans AC, Mcroberts RE, Lingner DV, Nicoletti AL, Moser P. Extensão original e atual da cobertura florestal de Santa Catarina. In: Vibrans AC, Sevegnani L, Gasper $\mathrm{AL}$, Lingner DV. Inventário florístico florestal de Santa Catarina: diversidade e conservação dos remanescentes florestais. Blumenau: Edifurb; 2012a.

Vibrans AC, Moser P, Maçaneiro JP, Lingner DV, Kruger A, Silva LS. Equações hipsométricas, volumétricas e de peso seco para a Floresta Ombrófila Densa em Santa Catarina. In: Vibrans AC, Sevegnani L, Gasper AL, Lingner DV. Inventário florístico florestal de Santa Catarina: Floresta Ombrófila Densa. Blumenau: Edifurb; 2012b.

Vismara EDS. Mensuração da biomassa e construção de modelos para construção de equações de biomassa [dissertação]. Piracicaba: Departamento de Engenharia Florestal, Universidade de São Paulo; 2009. 